\title{
The importance of clinical signs as well as radiological findings in the diagnosis of incomplete locked-in syndrome
}

\author{
Zeynep Özözen Ayas ${ }^{1}$, Dilcan Kotan², Aslı Aksoy Gündoğdu ${ }^{1}$
}

${ }^{1}$ Department of Neurology, Sakarya University Training and Research Hospital, Sakarya, Turkey

${ }^{2}$ Department of Neurology, Sakarya University Medical Faculty, Sakarya, Turkey

Submitted: 16 May 2016

Accepted: 15 June 2016

Arch Med Sci Atheroscler Dis 2016; 1: e60-e62

DOI: 10.5114/amsad.2016.60828

Copyright @ 2016 Termedia \& Banach

Incomplete locked-in syndrome is a rare syndrome of the brainstem in which, in contrast to the classical locked-in syndrome, some motor functions are not completely lost. This paper discusses the clinical presentation and magnetic resonance imaging (MRI) findings of a progressive incomplete locked-in syndrome in a 62-year-old man.

A 62-year-old, right-handed man presented to hospital with right-sided numbness for a few hours. His past medical history was notable for hypertension, diabetes mellitus, and compensated chronic renal disease, and a negative family history for any disorder. At the first hospital to which he was admitted, he was hypertensive at 260/130 $\mathrm{mm} \mathrm{Hg}$, for which intravenous glycerol trinitrate infusion was administered. He was then referred to our hospital. His neurological examination revealed hemihypoesthesia on the right side. He had a serum glucose level of $180 \mathrm{mg} / \mathrm{dl}$, urea $97 \mathrm{mg} / \mathrm{dl}$, and creatinine $2.8 \mathrm{mg} / \mathrm{dl}$. His electrocardiogram showed normal sinus rhythm. Brain tomography revealed no acute pathology. Brain diffusion weighted imaging (DWI) and apparent diffusion coefficient (ADC) MRI demonstrated a suspicious limitation in the left mesencephalon in only a single section (Figure 1). Blood pressure control was subsequently achieved and the patient was discharged from the hospital. However, he was re-admitted to the emergency department with reduced consciousness $5 \mathrm{~h}$ after his discharge. His vital signs were stable, whereas his neurological examination showed somnolence, limited response to verbal stimuli, and limited contraction on the right side. Further brain computed tomography (CT) did not reveal any acute pathology, but DWI and ADC MRI revealed an area of acute infarction in left mesencephalon (Figure 2). The patient was admitted to the intensive care unit and intubated, and antiaggregant treatment was started. During his course at the intensive care unit he was able to communicate. Later, he developed quadriplegia with preserved horizontal and vertical eye movements and slight cervical flexion. Repeat brain DWI and ADC MRI showed infarction with severe limitation of flow in the region of the left medial mesencephalon and pons (Figure 3). The patient currently receives intensive care for stroke.

In locked-in syndrome bilateral pontine lesions occur due to basilar artery thrombosis [1]. In this clinical condition consciousness is preserved but patients can only communicate by eye blinking and vertical eye movements. In 1979 Bauer et al. categorized this condition into three

\author{
Corresponding author: \\ Dilcan Kotan \\ Department of Neurology \\ Medical Faculty \\ Sakarya University \\ 25050 Sakarya, Turkey \\ Phone: 4882143030 \\ E-mail: dilcankotan@yahoo. \\ com
}



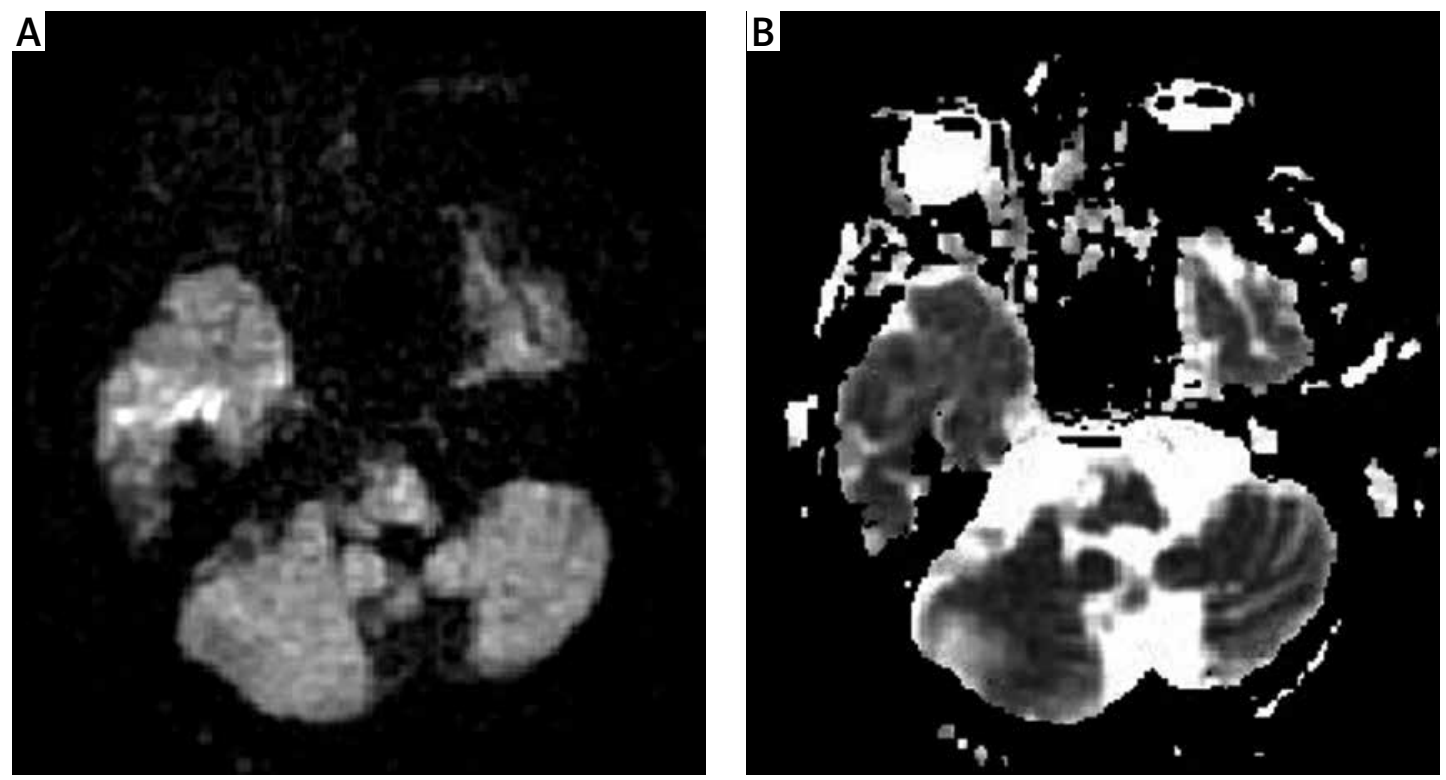

Figure 1 A, B. Brain DWI and ADC MRI showed a suspicious limitation in the left mesencephalon in only a single section
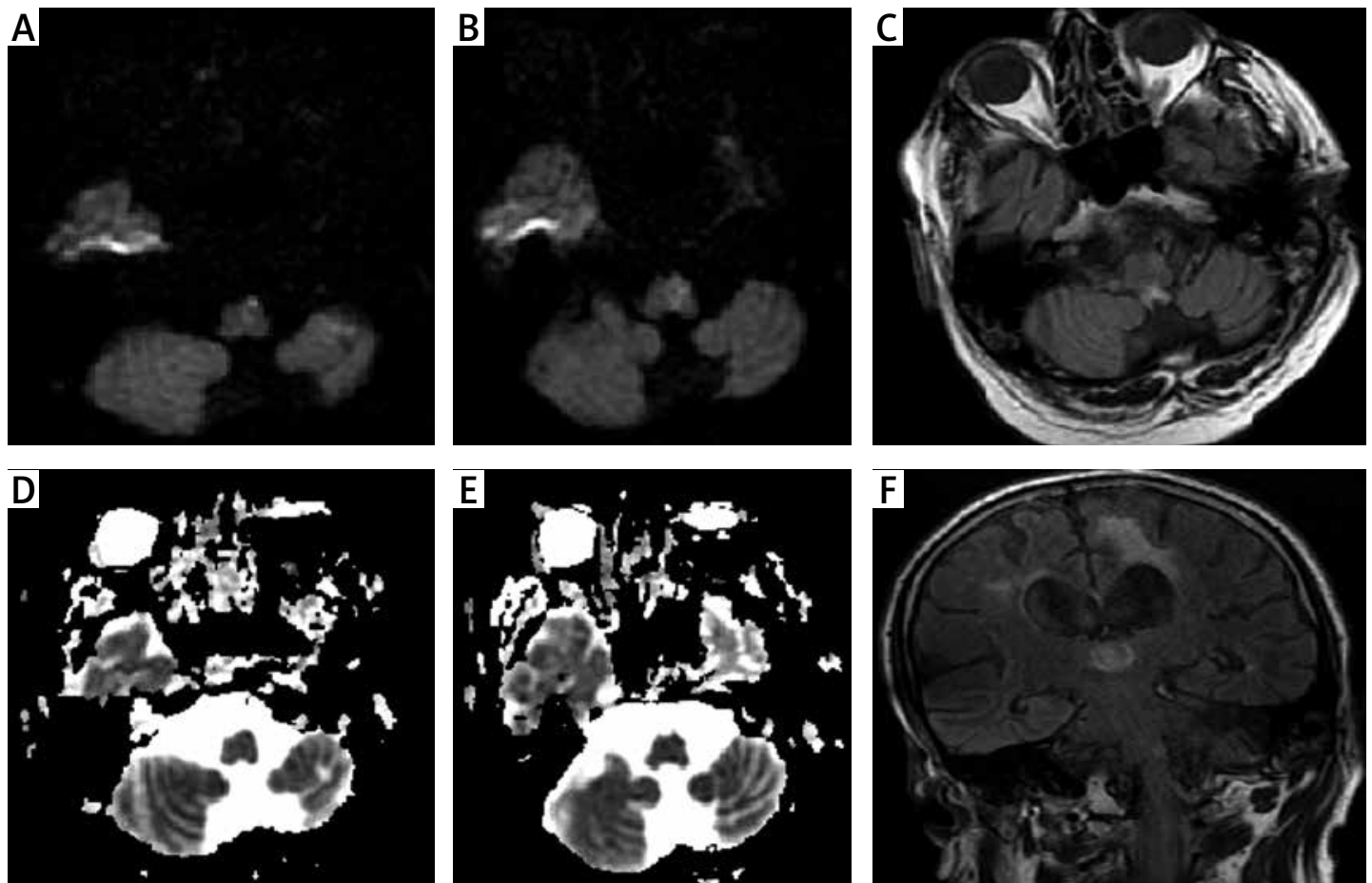

Figure 2 A-F. Brain DWI and ADC, FLAIR MRI revealed an area of acute infarction in the left mesencephalon

subtypes [2]. In the incomplete form, unlike the complete from, some motor functions are spared. In a series comprising 139 cases, 46 cases were categorized as incomplete forms [3]. Horizontal eye movements were preserved in 27 of them and fascial movements in 18 [3]. Our patient also had preserved cervical flexion in addition to horizontal and vertical eye movements. The patient could communicate by eye blinking and movements.

Our patient had only left hemihypoesthesia initially, but his symptoms deteriorated to incom- plete locked-in within several hours. Having a premorbid medical history of hypertension and diabetes, the patient had a full-blown disease picture within $24 \mathrm{~h}$, suggesting progressive thrombosis of the basilar artery as the culprit event. The prognosis of this condition is poor even at specialized stroke units unless the basilar artery can be recanalized early in the course [4]. Similar cases have been reported in the literature [5].

Progression of left-sided signs to quadriplegia was considered to be secondary to the compres- 

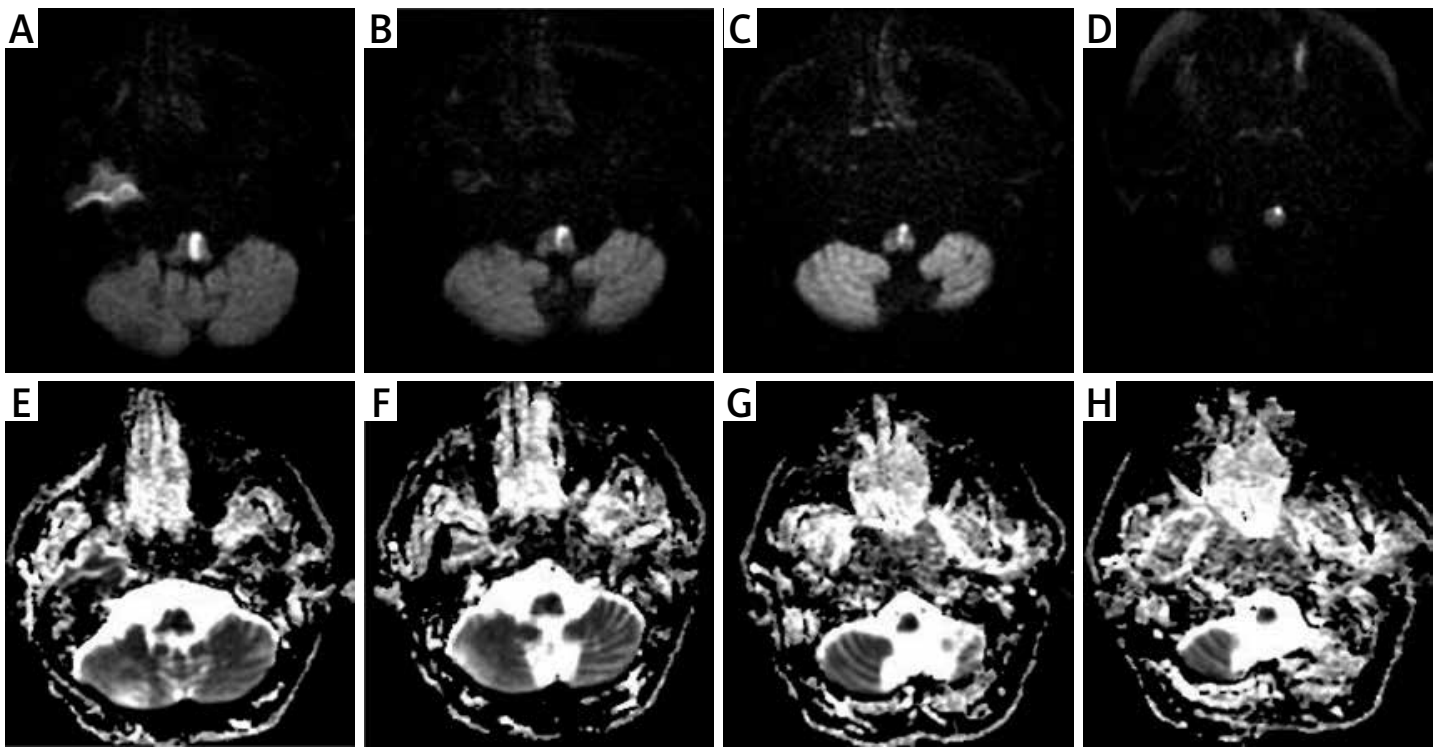

Figure $3 \mathrm{~A}-\mathrm{H}$. Brain DWI and ADC MRI showed infarction in the region of the left medial mesencephalon and pons

sion of the contralateral side by the medial involvement in the final MRI.

The brain stem is a site where image acquisition by neuroradiological studies is challenging. Brain CT can demonstrate limited pathological abnormalities in infarcted regions at early stages. DWI MRI is able to show areas of necrosis that exhibit cytotoxic edema beginning at the hyperacute stage [6]. For a true DWI limitation to occur, a reduction in ADC should occur. Pseudoappearances occur in the posterior fossa, and image resolution is reduced due to flow artefacts. Fluid attenuated inversion recovery (FLAIR) sequences have the advantage of fully suppressing cerebrospinal fluid signals. Our patient, who initially presented with a hypertensive state and left hemihypoesthesia, had an MRI study characterized by a diffusion limitation in a single section that had an unclear ADC counterpart, probably because of being taken very early in the course. Later it turned out, however, that conventional MRI sections could not be acquired due to technical reasons in that study. Selecting a correct size for image sections is of utmost importance too, particularly when assessing the brain stem. Nevertheless, as image acquisition is not performed with appropriate values in our center, it is possible that our patient's lesion may have been imaged in only a single section and missed. The patient had an acute infarction that can be shown by DWI, ADC, and FLAIR sequences of brain MRI, which was performed upon worsening of his overall status $5 \mathrm{~h}$ later.

A difficulty was experienced in our patient in establishing a correlation between the clinical and radiological findings. This study stresses the importance of obtaining conventional MRI sequences in addition to DWI and ADC MRI, particularly during hyperacute and acute periods of brainstem lesions; it also emphasizes the necessity of image acquisition under optimal conditions.

Recognition of locked-in syndrome is very important because of the predicted outcome. Although neuroimaging methods have been improved, clinical presentations should always be gold standard in this case. In this report, we aimed to evaluate the diagnostic limitations of diffusion-weighted magnetic resonance imaging in incomplete locked-in syndrome during the acute phase.

\section{Conflict of interest}

The authors declare no conflict of interest.

\section{References}

1. Plum F, Posner JB. The Diagnosis of Stupor and Coma. Philadelhia, Davis, 1966.

2. Bauer G, Gerstenbrand F, Rumpl E. Varieties of locked-in syndrome. J Neurol 1979; 221: 777-91.

3. Patterson JR, Grabios M. Locked-in syndrome: a review of 139 cases. Stroke 1986; 17: 758-64.

4. Vatankhah B, Gringel T, Stolze H. Acute basilar artery occlusion: need for an early diagnosis in a devastating disease. Stroke 2009; 40: e501.

5. Szilagyi T, Bajko Z, Liviu A, Szabolcs S. Incomplete locked-in syndrome. Rom J Neurol 2011; 10: 94-7.

6. Marks MP, de Crespigny AJ, Lentz D, Enzmann DR, Albers GW, Moseley ME. Acute and chronic stroke: navigated spin-echo diffusion-weighted MR imaging. Radiology 1996; 199: 403-8. 